Rademakers, J.J.D.J.M., Muijsenbergh, M.E.T.C. van den, Slappendel, G., Lagro-Janssen, A.L.M., Borleffs, J.C.C. Sexual harassment during clinical clerkships in Dutch medical schools. Medical Education: 2008, 42(5), 452-458

\begin{tabular}{|l|l|}
\hline Postprint Version & 1.0 \\
\hline Journal website & $\mathrm{http} / /$ www.blackwell-synergy.com/doi/abs/10.1111/j.1365-2923.2007.02935.x \\
\hline Pubmed link & $\begin{array}{c}\text { http://www.ncbi.nlm.nih.gov/entrez/query.fcgi?cmd=retrieve\&db=pubmed\&do } \\
\text { pt=Abstract\&list_uids=18346119 }\end{array}$ \\
\hline DOI & $10.1111 / \mathrm{j} .1365-2923.2008 .03016 . x$ \\
\hline
\end{tabular}

This is a NIVEL certified Post Print, more info at http://www.nivel.eu

\title{
Sexual harassment during clinical clerkships in Dutch medical schools.
}

\author{
RADEMAKERS, J.J.D.J.M., MuIJSENBERGH. M.E.T.C. VAN DEN, SLAPPENDEL, G., LAGRO- \\ JANSSEN, A.L.M., BORLEFFS, J.C.C. \\ Dr J.J.D.J.M. Rademakers MA PhD, psychologist, associate professor at UMC Utrecht, \\ School of Medical Sciences, Center for Research and Development of Education. \\ Dr. M.E.T.C. van den Muijsenbergh MD PhD GP, Department of General Practice, Women's \\ Studies, Medical Sciences UMC Radboud University Nijmegen \\ Ms G. Slappendel, graduate student Psychology, research assistant at UMC Utrecht, School \\ of Medical Sciences, Center for Research and Development of Education. \\ Prof. Dr A.L.M. Lagro-Janssen MD PhD GP, Department of General Practice, Women's \\ Studies, Medical Sciences UMC Radboud University Nijmegen \\ Prof. Dr J.C.C. Borleffs MD PhD, professor of medical education, director of medical \\ programs at UMC Utrecht, School of Medical Sciences, and Department of Internal \\ Medicine.
}

\begin{abstract}
\section{BACKGROUND}

Sexual harassment of medical students has been the focus of many international studies. In these studies prevalence rates from 18 to over $60 \%$ were reported. In a Dutch study at Nijmegen medical school, however, the prevalence rate was lower (13.3\% total group; $20 \%$ female students only).
\end{abstract}

AIM

Identify whether Nijmegen constitutes a positive sample of Dutch medical schools or whether incidents of sexual harassment are less prevalent in the Netherlands altogether; Establish if and how these experiences impact the (professional) lives of students.

\section{METHODS}

Students received a semi-structured questionnaire with questions about their experiences with sexual harassment during clerkships. The reaction to the incident, possible consequences for well-being and/or professional functioning and the way the case was handled were questioned.

\section{RESULTS}

The prevalence of sexual harassment is significantly higher in Utrecht as compared to Nijmegen. In both studies the rates were relatively low compared to international data. Nevertheless, one in three to five Dutch female medical 
Rademakers, J.J.D.J.M., Muijsenbergh, M.E.T.C. van den, Slappendel, G., Lagro-Janssen, A.L.M., Borleffs, J.C.C. Sexual harassment during clinical clerkships in Dutch medical schools. Medical Education: 2008, 42(5), 452-458

students experience unwelcome sexually-coloured attention by patients, colleagues or even supervisors. Three out of ten students who experienced such an incident state it had a negative impact on their functioning afterwards.

\section{CONCLUSION}

The prevalence rates of sexual harassment in medical schools is the Netherlands is low compared to international rates. On the other hand the number of women students who experience sexual harassment is still one in three to five. The occurrence of and dealing with these incidents should be an important topic in the training of medical students and supervisors.

\section{INTRODUCTION}

Since the early nineties, sexual harassment of medical students has become the focus of many studies throughout the Western world ${ }^{1-14}$. Most studies have been done in the United States, Canada, Australia, and Scandinavia.

Prevalence rates vary greatly (from 18 to over $60 \%$ ) and depend on the definition of sexual harassment, the report period and the research methodology (f.e. prompting or not). Causes of the relative high prevalence rates in medical schools include the male-dominated culture, with its hierarchical structure and conservative elements. Research has demonstrated that these factors facilitate the occurrence of sexual harassment ${ }^{15-17}$. Furthermore, two categories of offenders are possible for medical students: patients and medical doctors/colleagues.

Sexual harassment by patients is unexpected: one would think that the role of medical doctor protects students from sexual harassment by patients (who are to an important extent dependent on their doctors). But for female doctors and medical students, it does not: the traditional power of men over women seems to compensate for this ${ }^{18}$.

Sexual harassment has a negative impact on students' emotional and physical well-being and on their professional behaviour ${ }^{4911}$. The effect depends on the severity of the incident and on the characteristics of the offender. Students might feel guilty or ashamed, and some feel frightened or inhibited in their contacts with patients afterwards. Sexual harassment may lead to diminished work satisfaction and sick leave.

Until 2003, no study was done in the Netherlands with regard to the prevalence, type and consequences of sexual harassment of medical students. In that year the University Medical Center St. Radboud in Nijmegen collected data among their $5^{\text {th }}$ and $6^{\text {th }}$ year students about sexual harassment during their clinical clerkships ${ }^{19}$. Clinical clerkships are the setting in which students have encounters with real patients and therefore the ideal situation for studying aspects of clinical interaction (including possible incidents of sexual harassment). Sexual harassment -in accordance with Dutch law- was defined as unwelcome, sexuallycoloured attention. In this definition, the emphasis is on how people experience certain remarks and behaviours, not on how they are intended. One of the results of this study was a prevalence rate of $13.3 \%$ of the total group and $20 \%$ among female students only. Although one fifth of all female students experiencing sexual harassment is not a small percentage, this prevalence rate is relatively low compared to other studies. This led to the question whether this medical school constitutes a positive sample of Dutch medical schools or whether incidents of sexual harassment are less prevalent in the Netherlands altogether. Therefore, in 2005 - 2006 this study was replicated in the University Medical Center of Utrecht. This study was approved by the Medical Ethical Committee of the UMC Utrecht.

As the Netherlands are a relatively small country, differences between the student populations of the medical schools are considered to be minimal. The schools are all state funded, and though students may express their preferred choice for a certain medical school they are assigned to one by computer. Both schools in this study are located outside the 
Rademakers, J.J.D.J.M., Muijsenbergh, M.E.T.C. van den, Slappendel, G., Lagro-Janssen, A.L.M., Borleffs, J.C.C. Sexual harassment during clinical clerkships in Dutch medical schools. Medical Education: 2008, 42(5), 452-458

bigger, multicultural cities (Amsterdam and Rotterdam). They only differ with respect to aspects of the curriculum. Students in Utrecht start earlier with clerkships compared to Nijmegen. In both schools guidelines and policies concerning the education about and reporting of sexual harassment are similar. In this article, the data from Utrecht and Nijmegen are compared to each other.

\section{METHOD}

In both University Medical Centers medical students received the same semi-structured questionnaire. The questionnaire was developed for the Nijmegen study on the basis of a literature study. The questionnaire consisted of three parts. The first part were questions on general demographic and other background variables. The second contained 13 questions about experiences students might have had with sexual harassment during clerkships (and if so, a qualitative description of the most severe incident). Sexual harassment was defined as unwelcome, sexually-coloured attention. The reaction of the student to this incident, consequences for their well-being and/or professional functioning and the way the case was handled were asked. The third part (for students who had no experiences to report) was about these students' perspectives on possible determinants of sexual harassment during clerkships. In Utrecht, the questionnaire was handed out by the researchers to $6^{\text {th }}$ year medical students $(\mathrm{N}=141)$ during an important introductory lecture at the start of their $6^{\text {th }}$ year. Though we do not know the exact male / female representation at this lecture, in the total year group $(\mathrm{N}=$ 260 ), the percentages were $28,5 \%$ male and $71,5 \%$ female. The questionnaires were collected at next day's lecture. In Nijmegen, the questionnaire was handed out by the researchers to one full year group of interns $(\mathrm{N}=183)$ who could be either $5^{\text {th }}$ or $6^{\text {th }}$ year medical students, during three days on which the interns return to the medical school. Of these $183,38,3 \%$ were male and $61,7 \%$ were female students. They were asked to send the questionnaires back by mail (a return envelope was included).

The results from these questionnaires were analysed with SPSS, using $\mathrm{X}^{2}$-tests to establish significance. Differences at a level $<.05$ were considered significant. The qualitative information was coded and categorized.

\section{RESULTS}

The response rate in Utrecht was $81 \%(\mathrm{~N}=114)$ of which $23.9 \%$ was male and $76.1 \%$ female. The mean age was 24.5 years. The response rate in Nijmegen was $66 \%(\mathrm{~N}=113)$ of which $33.6 \%$ was male and $66.4 \%$ female. The mean age was 24 years. The difference in response rate might be due to the fact that Nijmegen students were supposed to return the questionnaire by mail themselves, where in Utrecht the researcher came back the next day to collect them.

\section{Prevalence of sexual harassment}

Of the total group, one in five students reports to have been sexually harassed (table 1). In Utrecht the prevalence is significantly higher as compared to Nijmegen $(\mathrm{X} 2=6,987$; $\mathrm{df}=1$; $\mathrm{p}=.008)$.

\section{[TABLE 1]}

Sexual harassment is almost exclusively a problem with which female students are confronted. In Utrecht, one male student reported to have been sexually harassed, while in Nijmegen only female students reported such experiences. When the data are analyzed for female students only, the prevalence rates rise to $28.1 \%$ in the total group, $34.9 \%$ in Utrecht and $20.0 \%$ in Nijmegen. As in the total group, this difference between the two medical schools is statistically significant $\left(X^{2}=4,610 ; d f=1 ; p=.032\right)$. 
Rademakers, J.J.D.J.M., Muijsenbergh, M.E.T.C. van den, Slappendel, G., Lagro-Janssen, A.L.M., Borleffs, J.C.C. Sexual harassment during clinical clerkships in Dutch medical schools. Medical Education: 2008, 42(5), 452-458

The number of experiences per student did not differ significantly between the groups $\left(X^{2}=7,126 ; d f=1 ; p=.129\right)$ : the majority of the respondents had experienced one incident of sexual harassment (Utrecht $66.7 \%$; Nijmegen $60.0 \%$ ). In both cities, one out of 15 students $(6.7 \%)$ reported more than three incidents.

Students were asked to describe the most severe incident they had experienced. In total, 45 incidents of sexual harassment were described in more detail. In figure 1, the different types of sexual harassment are shown. During one incident more than one type of harassment may occur, therefore the percentages in this figure add up to over $100 \%$.

\section{[FIGURE 1]}

Most of the incidents in both medical schools referred to flirtatious or sexual remarks. 'The patient kept on saying that he would wait for me later, that I was such a dishy little doctor.'

'The patient repeatedly asked me whether we were going to have sex. We were alone in the consulting room were I did a physical examination. Nobody else was present.'

'The patient said that he liked to be helped by tasty women and if I wanted to give him some extra 'special' attention.'

The second most prevalent category is that of 'other unwanted behaviour'. This category is diverse but includes situations where the patient undresses (much) more than necessary or appropriate for the physical examination.

'I had to examine his arms, but he insisted in taking his trousers off. We were alone in a consulting room. Nobody else was there. I have told him a few times that I didn't think that was necessary, until he stopped.'

'When the patient had to undress for his physical examination, he had undressed totally and was suddenly sitting naked in front of me. And I had explicitly told him that he could keep his underwear on.'

Another example of unwanted behaviour is:

'A patient slapped me with a folded newspaper two times on my behind and said 'Young doctors nowadays'. I was busy with another patient so I was stunned and slightly irritated.'

Sometimes the patient actually gets physically aroused from the physical examination. Female students might feel uncomfortable when this happens and either ignore it or try to finish the examination as quickly as possible.

Personal questions seem much more common in Utrecht than in Nijmegen:

'The surgeon asked about my boyfriend and my sex life. At the same time he pressed himself against me, supposedly because he had to reach for something.'

'The patient asked me how old I was, and said I was a very beautiful young doctor.'

The most severe form of sexual harassment is the one where there is actual, unwanted, physical contact.

'I had to take blood from a patient. He started touching my leg while I took blood. We were alone in his room. I had to finish the blood taking first and then I left quickly after I had shaken his hand off my leg.'

'I wanted to ask a question about a patient I had just seen. We were on the ward and I took something out of a cupboard. Then he (the doctor) stood right behind me, put his hands on my side, turned me around so I stood between him and the cupboard.. I felt very uneasy then.'

'He (the patient) made remarks about my looks, asked provocative questions, made a little physical contact. I commented on it and I refused to do a physical examination.'

\section{Types of offenders}

In all but one case, the offenders were male. Two thirds were patients; in almost one third medical doctors (or residents) were involved in the incident (table 2). They might have been colleagues (a medical doctor or resident who worked in the same department but who did not 
Rademakers, J.J.D.J.M., Muijsenbergh, M.E.T.C. van den, Slappendel, G., Lagro-Janssen, A.L.M., Borleffs, J.C.C. Sexual harassment during clinical clerkships in Dutch medical schools. Medical Education: 2008, 42(5), 452-458

have specific supervision tasks), but also two incidents with supervisors have been reported. Students did not report incidents with fellow-students. There were no statistically significant differences between the two medical schools $\left(X^{2}=2,829 ; \mathrm{df}=3 ; \mathrm{p}=.419\right)$.

\section{[TABLE 2]}

In both schools, the incidents predominantly happened during the clerkships internal medicine, psychiatry and neurology. In Nijmegen, incidents during general practice clerkship were reported as well, and in Utrecht during surgery clerkships. In general, there is no significant difference between the schools $\left(X^{2}=12,575 ; \mathrm{df}=7 ; \mathrm{p}=.083\right)$ with respect to the clerkships in which the incidents occurred. Sexual harassment by patients is distributed quite evenly across the clerkships.

\section{Impact of the incident and support afterwards}

Three out of ten students (29.5\%) who experienced sexual harassment state it had a negative impact on their functioning afterwards. In Nijmegen $46.7 \%$ of the students stated the experience affected their professional conduct, while in Utrecht $20.0 \%$ of the students said so $\left(X^{2}=3.462 ; d f=1 ; p=.063\right)$. Students doubt their own judgments whether certain behaviour should be considered (in)appropriate, especially when other colleagues do not seem to regard the incidents as problematic.

'It influenced my functioning during the clerkship, the relationship with that doctor and the nurses, that they did not take it seriously...They thought everything was normal.'

Some students feel uncertain and inhibited when making contact with patients after the incidents.

Though the numbers are too small for statistical analysis, the impact of the incident seems bigger when the offender is a medical doctor or resident. Students who experienced harassment by medical doctors more often say it had impact on their subsequent behaviour and more often discussed the incidents with others.

The majority of students who experienced sexual harassment discuss the incident with others $(77.8 \%)$, usually peers. There is no significant difference between the two medical schools in this respect. In Nijmegen, students more often tend to confront the offender with his unwanted behaviour ( $46.7 \%$ vs. $20.0 \%$ in Utrecht; $\left(X^{2}=3.462\right.$; $\left.d f=1 ; p=.063\right)$. Threequarters $(73.3 \%)$ of the students are satisfied with the way the incident has been dealt with, but one in four $(26.7 \%)$ was not. Some of them would have wanted to confront the offender with the inappropriateness of his behaviour, but had not done so. Others were not happy with their supervisors' reaction to the incident, or with the fact that sexual harassment seemed to be accepted as normal behaviour in certain departments. Of the total group, $13.3 \%$ experienced a lack of special support afterwards. The medical schools did not differ in this respect.

\section{DISCUSSION AND CONCLUSIONS}

In this study in two Dutch medical schools, one in five medical students reports unwelcome sexually-coloured attention by patients, colleagues or even supervisors. The prevalence in one school (Utrecht) is significantly higher compared to the other (Nijmegen). Sample composition could have had an effect on this difference in prevalence: the samples differed with respect to gender and whether or not $5^{\text {th }}$ year students were included. The Nijmegen sample contained more male respondents than the Utrecht sample (33.6\% vs. $23.9 \%)$, though this difference is not significant. Males are far less likely to be the object of sexual harassment. However, when the analysis was done for female students only, the prevalence rates between the schools remained significantly different. The inclusion of $5^{\text {th }}$ year students in Nijmegen might have lowered the prevalence in that sample: since those students probably have not finished all their year 5 clerkships yet, they had less exposure in this respect. Though we have no exact data on the number of weeks and months the respondents spent in 
Rademakers, J.J.D.J.M., Muijsenbergh, M.E.T.C. van den, Slappendel, G., Lagro-Janssen, A.L.M., Borleffs, J.C.C. Sexual harassment during clinical clerkships in Dutch medical schools. Medical Education: 2008, 42(5), 452-458

clerkships, the fact that on average the Utrecht respondents are a half year older than the Nijmegen students confirms the idea that they have more clinical experience. Also, the fact that Utrecht students start earlier with their clerkships supports this explanation. However, it was not feasible to recruit the students at exactly the same moment in both schools due to differences in the organization of the curriculum.

Three out of ten students who experienced an incident of sexual harassment state it had a negative impact on their functioning afterwards. This may lead to diminished work satisfaction on the part of the student, but also to a loss of quality of care since students feel afraid or inhibited in their contacts with patients.

The impact of sexual harassment seems even bigger when the offender is a medical doctor or a resident. Female students seem to be confronted with an extra task in their professional development and career: not only do they have to learn to behave professionally themselves, they also have to learn to deal with colleagues who do not. The individual or collective (e.g. departmental) acceptance of sexual harassment reflects the traditional hierarchical, maledominated culture which seems to persevere in certain (teaching) hospitals or departments. The fact that women would not want to work in such an atmosphere could restrict their specialization and career choices.

One in four students is not satisfied with the way the incident was handled, and one in eight experienced a lack of special support after the incident. It is clear that the occurrence of and how to deal with these incidents should be an important topic in the training of medical students and their supervisors. This should take place in communication and attitude training (for students) and in teacher development sessions. Furthermore formal complaint procedures should be better known and more easily accessible to students. Students who have experienced an incident of sexual harassment should feel supported by their supervisors and the medical school in general. An information and prevention campaign could be part of transferring this message to students.

One of the questions we wanted to answer with this study is whether incidents of sexual harassment are less prevalent in the Netherlands compared to other countries. Though we consider a prevalence rate of $20.4 \%$ in the total group and $28.1 \%$ among female students very high, compared to data from international literature as presented in the introduction of this paper (18-60\%) the Dutch rates are within the lower segments. This may imply that the behaviour itself is either less prevalent in the Netherlands, or that Dutch medical students (or young women in general) will be more able to deal with this type of behaviour themselves and will not consider some incidents important enough to report them as harassment. The first hypothesis regarding the lower prevalence rates is substantiated by research by Hofstede ${ }^{20}$ who researched masculinity versus femininity as a cultural aspect in different societies. He concluded that in more masculine societies like the United States differences between men's and women's roles are more prominent and people are more traditional and prudish about sexuality. Sexual behaviour is more gendered in a masculine culture, and the risk of sexual harassment increases in a culture where men have to 'prove' their masculinity while women are supposed to be passive and (when unmarried) preferably virgins. The Netherlands, on the other hand, is considered one of the most feminine cultures in the world in which gender-equality in social and sexual interaction is more pervasive. Other international comparative studies ${ }^{21-23}$ confirm the second hypothesis of Dutch women and girls being more assertive and able to express their boundaries. These studies have shown that open attitudes towards sexuality in a society and comprehensive sexual education, like in the Netherlands, have a positive effect on sexual health behaviour because it empowers young men and women and gives them the skills to communicate and negotiate on sexuality.

Because our study shows that there are differences with respect to the incidence of sexual harassment between medical schools in one country and between clinical specialties in hospitals, comparative qualitative studies on cultural differences in these specific settings 
Rademakers, J.J.D.J.M., Muijsenbergh, M.E.T.C. van den, Slappendel, G., Lagro-Janssen, A.L.M., Borleffs, J.C.C. Sexual harassment during clinical clerkships in Dutch medical schools. Medical Education: 2008, 42(5), 452-458

could give more insight in the determinants and characteristics of sexual harassment. Masculinity and femininity might also be important cultural aspects at micro level (organization, ward). It might have impact on the definition of which behaviour is considered to be sexual harassment (and which is not). Also, the expectations of students, colleagues, and patients with respect to the professional encounter (e.g. are personal questions and compliments about appearance allowed or not?) are important. From other studies we already know that expectations from patients seem to be 'gendered', meaning that patients expect different kinds of behaviour from male compared to female doctors ${ }^{24,25}$. To gain further insight in the determinants of sexual harassment of medical students on a macro level, international comparative research would be helpful.

\section{ACKNOWLEDGEMENTS}

The authors would like to thank Ms M.A. Kruijshoop, MA, who made valuable suggestions on improving the article with respect to grammar and style.

\section{REFERENCES}

${ }^{1}$ Elnicki DM, Linger B, Asch E, Curry R, Fagan M, Jacobson E, et al. Patterns of medical student abuse during the internal medicine clerkship: perspectives of students at 11 medical schools. Acad Med 1999;74(10 Suppl):S99-101.

2 Kassebaum DG, Cutler ER. On the culture of student abuse in medical school. Acad Med 1998;73;1149-58.

${ }^{3}$ Larsson C, Hensing G, Allebeck P. Sexual and gender-related harassment in medical education and research training: results from a Swedish survey. Med Educ 2003;37:39-50.

${ }^{4}$ Lubitz RM, Nguyen DD. Medical student abuse during third-year clerkship. JAMA 1996; 275:414-16.

${ }^{5}$ Moscarello R, Margittai KJ,Rossi M. Differences in abuse reported by female and male Canadian medical students. CMAJ 1994;150:357-63.

${ }^{6}$ Nora LM. Sexual harassment in medical education: a review of the literature with comments from the law. Acad Med 1996;71(1 Suppl):S113-8.

${ }^{7}$ Nora LM, McLaughlin MA, Fosson SE, Stratton TD, Murphy-Spencer A, Fincher RM, et al. Gender discrimination and sexual harassment in medical education: perspectives gained by a 14-school study. Acad Med 2002;77:1226-34.

${ }^{8}$ Richardson DA, Becker M, Frank RR, Sokol RJ. Assessing medical students' perception of mistreatment in their second and third years. Acad Med 1997;72:728-30.

${ }^{9}$ Richman JA, Flaherty JA, Rospenda KM, Christensen ML. Mental health consequences and correlates of reported medical student abuse. JAMA 1992;267:692-4.

${ }^{10}$ Sheehan KH, Sheehan DV, White K, Leibowitz A, Baldwin jr DC. A pilot study of medical student 'abuse'. Student perceptions of mistreatment and misconduct in medical school. JAMA 1990;263:533-7.

${ }^{11}$ Silver HK, Glicken AD. Medical student abuse. Incidence, severity and significance. JAMA 1990;263:527-32.

${ }^{12}$ Uhari M., Kokkonen J, Nuutinen M, Vainionpaa L, Rantala H, Lautala P, et al. Medical student abuse: an international phenomenon. JAMA 1994;271:1049-51.

${ }^{13}$ White GE. Sexual harassment during medical training: the perception of medical students at a university medical school in Australia. Med Educ 2000;34:980-6.

${ }^{14}$ Wolf TM, Randall HM, Almen K von, Tynes LL. Perceived mistreatment and attitude change by graduating medical students: a retrospective study. Med Educ 1991;25:182-90.

${ }^{15}$ Gruber JE. The impact of male work environments and organizational policies on womens' experiences of sexual harassment. Gend Soc 1998;12:301-20.

${ }^{16}$ Luthar HK, Pastille C. Modeling subordinate perceptions of sexual harassment: the role of superior-subordinate social-sexual interaction. Hum Resour Manage 2000;10:211-44.

${ }^{17}$ O'Hare EA, O'Donohue W. Sexual harassment: identifying risk factors. Arch Sex Behav 1998;27:561-80.

${ }^{18}$ Schneider M, Phillips SP. A qualitative study of sexual harassment of female doctors by patients. Soc Sci Med 1997;45:669-76. 
Rademakers, J.J.D.J.M., Muijsenbergh, M.E.T.C. van den, Slappendel, G., Lagro-Janssen, A.L.M., Borleffs, J.C.C. Sexual harassment during clinical clerkships in Dutch medical schools. Medical Education: 2008, 42(5), 452-458

${ }^{19}$ Muijsenbergh METC van den , Lagro-Janssen ALM. Sexual harassment of medical students during their period of work placement. Ned Tijdschr Geneeskd 2005;149 (14):7648 (in Dutch)

${ }^{20}$ Hofstede G and associates. Masculinity and Femininity: The Taboo Dimension of National Cultures. Thousand Oaks CA: Sage Publications, 1998.

${ }^{21}$ Jones $\mathrm{E}$. et al. Teenage pregnancy in industrialized countries: determinants and policy implications. Fam Plann Perspect. 1985 Mar-Apr;17(2):53-63

${ }^{22}$ Ferguson RM. Comparison between sexuality education in the Netherlands and United States (in press)

${ }^{23}$ Brugman MJE. Emerging adolescent sexuality: a comparison of American and Dutch women's experiences. PhD-thesis, Graduate School, University of Maine (2007).

${ }^{24}$ Schmid Mast M. Dominance and gender in the physician-patient interaction. Journal of Men's Health \& Gender, 2004; 1:354-58.

${ }^{25}$ Kilminster S, Downes J, Gough B, Murdoch-Eaton D, Roberts T. Women in medicine - is there a problem? A literature review of the changing gender composition, structures and occupational cultures in medicine. Medical Education, 41, 2007, 39-49.

\section{TABLES AND FIGURES}

Table 1: Experience with sexual harassment (in \%), total group

\begin{tabular}{|l|cl|ll|ll|}
\hline & \multicolumn{2}{|c|}{ Utrecht } & \multicolumn{2}{|l|}{ Nijmegen } & \multicolumn{2}{l|}{ Total } \\
& $\begin{array}{l}\text { Total Female } \\
\text { sample students }\end{array}$ & $\begin{array}{l}\text { Total Female } \\
\text { sample students }\end{array}$ & $\begin{array}{l}\text { Total Female } \\
\text { sample students }\end{array}$ \\
\hline Yes & 27.4 & 34.9 & 13.3 & 20.0 & 20.4 & 28.1 \\
\hline No & 72.6 & 65.1 & 86.7 & 80.0 & 79.6 & 71.9 \\
\hline $\mathrm{N}=$ & 114 & 87 & 113 & 75 & 227 & 162 \\
\hline
\end{tabular}

Table 2: Types of offenders (in \%), total group

\begin{tabular}{|llll|}
\hline & Utrecht & Nijmegen & Total \\
Patients & $71.0(\mathrm{~N}=21)$ & $60.0(\mathrm{~N}=9)$ & $67.4(\mathrm{~N}=30)$ \\
Doctors & $29.0(\mathrm{~N}=9)$ & $40.0(\mathrm{~N}=6)$ & $32.6(\mathrm{~N}=15)$ \\
\hline
\end{tabular}


Rademakers, J.J.D.J.M., Muijsenbergh, M.E.T.C. van den, Slappendel, G., Lagro-Janssen, A.L.M., Borleffs, J.C.C. Sexual harassment during clinical clerkships in Dutch medical schools. Medical Education: 2008, 42(5), 452-458

Figure 1: Sexual harassment incidents $(\mathrm{N}=45)$ by type of behaviour $(\%$ of respondents reporting such behaviour)

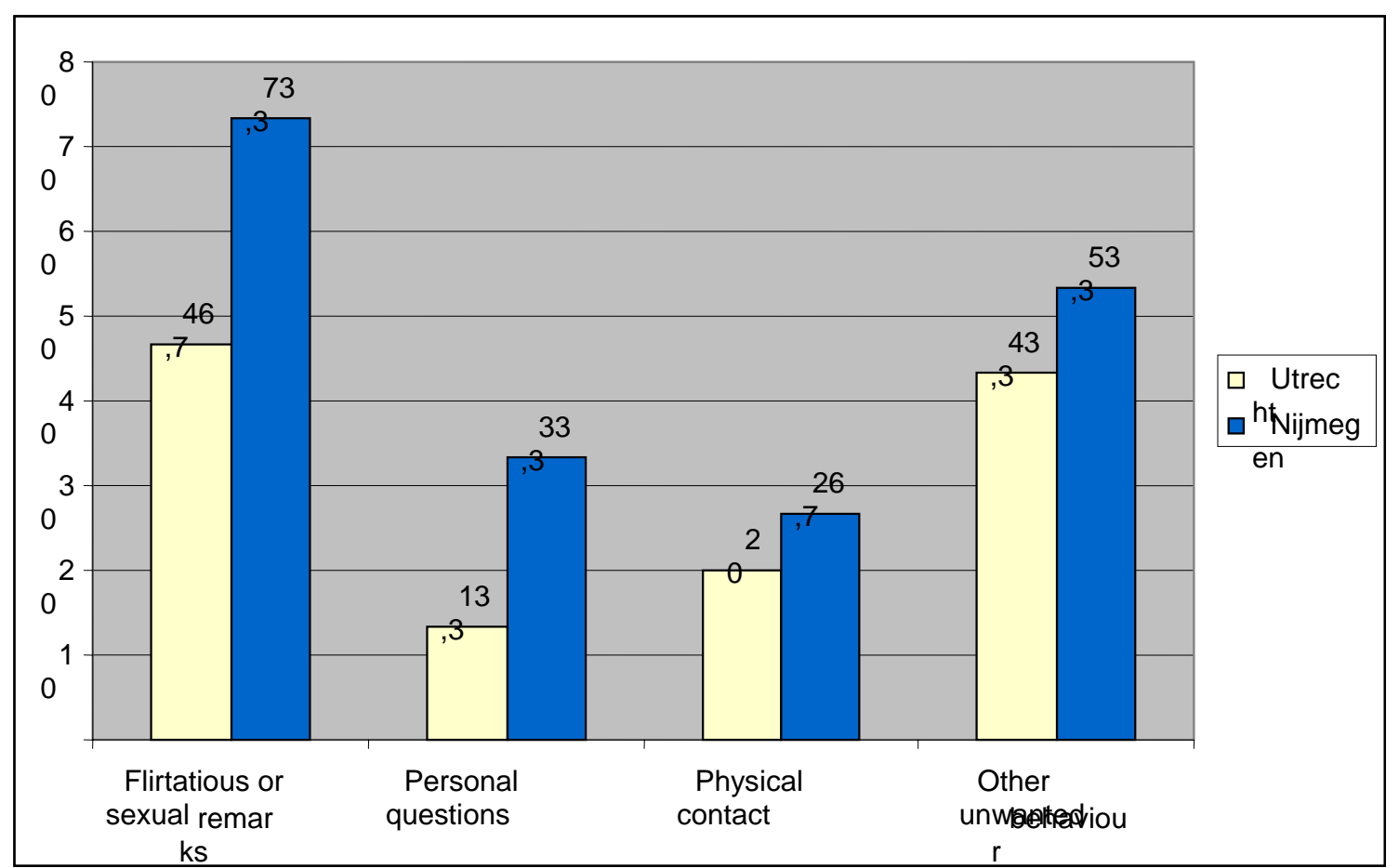

\section{Overview box}

What is already known about this subject?

- Many international studies have been done focusing of sexual harassment of medical students. These studies show prevalence rates from $18 \%$ to over $60 \%$. The first Dutch study (done at Nijmegen medical school in 2003) showed lower prevalence rates.

- Sexual harassment has a negative impact on the on emotional and physical wellbeing and on professional behaviour. Students might feel guilty or ashamed, some feel frightened or inhibited in their contacts with patients afterwards. Sexual harassment can lead to diminished work satisfaction and sick leave.

What this study adds?

- This study confirms that harassment by medical doctors or residents has a more negative impact on students.

- Incidents predominantly happened during the clerkships internal medicine, psychiatry and neurology. 
Rademakers, J.J.D.J.M., Muijsenbergh, M.E.T.C. van den, Slappendel, G., Lagro-Janssen, A.L.M., Borleffs, J.C.C. Sexual harassment during clinical clerkships in Dutch medical schools. Medical Education: 2008, 42(5), 452-458

- Though we consider a prevalence rate of $20.4 \%$ in the total group and $28.1 \%$ among female students very high, compared to data from international literature the Dutch rates are within the lower segments.

Suggestions for further research

- Qualitative studies which respect to cultural differences between medical schools and between clinical specialties could give information on both risk factors and protective factors for the occurrence of sexual harassment in a specific setting.

- To gain further insight in the determinants of sexual harassment of medical students, international comparative research would be helpful. 